\title{
Early radiographic and functional outcomes of a cancellous titanium-coated tibial component for total knee arthroplasty
}

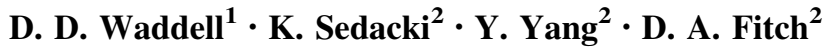

Received: 30 April 2015/Accepted: 7 September 2015/Published online: 26 September 2015

(c) The Author(s) 2015. This article is published with open access at Springerlink.com

\begin{abstract}
Background Various surface coatings have been developed over the past decades to enhance fixation of cementless total knee arthroplasty (TKA). BIOFOAM ${ }^{\circledR}$ (MicroPort Orthopedics Inc., Arlington, TN, USA) is a novel cancellous titanium surface coating intended to increase both initial and long-term fixation. The purpose of this study was to investigate the early functional and radiographic outcomes of this coating used in a TKA application.

Materials and methods One hundred and four (104) primary TKAs in 85 subjects using BIOFOAM-coated tibial components were prospectively enrolled at four centers. Subjects were evaluated using Knee Society Scores and radiographic analysis at a minimum follow-up of 24 months.

Results Knee Society Scores and flexion were all significantly improved at final follow-up compared to baseline. Radiographic analyses were satisfactory, with no progressive radiolucencies and only a single subject presenting with a radiolucency surrounding a tibial component. There were two revisions in the cohort: one for instability following a ruptured lateral collateral ligament and one for recurrent tibial insert dislocation.

Conclusions This is the first study to report clinical outcomes associated with the BIOFOAM coating used in a cementless TKA application. Early functional scores and radiographic analyses are promising, but further
\end{abstract}

D. A. Fitch

david.fitch@ortho.microport.com

Orthopedic Specialists of Louisiana, Shreveport, LA, USA

2 MicroPort Orthopedics Inc., 5677 Airline Rd., Arlington, TN 38002, USA investigations are needed to confirm long-term clinical success with these components.

Keywords Total knee arthroplasty - Total knee replacement · BIOFOAM - Cementless fixation . ADVANCE

\section{Introduction}

The ADVANCE ${ }^{\circledR}$ Medial-Pivot System (MicroPort Orthopedics Inc., Arlington, TN, USA) was first introduced in 1998 by Wright Medical Technology, Inc. The cemented version of this total knee arthroplasty (TKA) system has been shown to have a long, satisfactory clinical history [1-6]. The system was later expanded to include titanium porous bead-coated components for cementless or hybrid fixation, and eventually a tibial component featuring a cancellous titanium coating commercially called BIOFOAM $^{\circledR}$ (Fig. 1).

BIOFOAM is a porous reticulated titanium material developed for load-bearing orthopedic applications and features a compressive modulus similar to that of native bone [7]. This is significant as differences in mechanical properties between native bone and implants can lead to stress shielding resulting in radiolucencies and decreased bone mineral density. A recent study found evidence of this occurring in TKAs performed using both cemented and titanium porous bead cementless fixation [8]. BIOFOAM also has other material properties (e.g., increased porosity and coefficient of friction) that make it attractive for the use in cementless TKA, but clinical outcomes have not yet been reported for this application. The objective of this preliminary study was to evaluate the short-term radiographic and functional outcomes for subjects implanted with BIOFOAM-coated tibial components used for cementless TKA. 


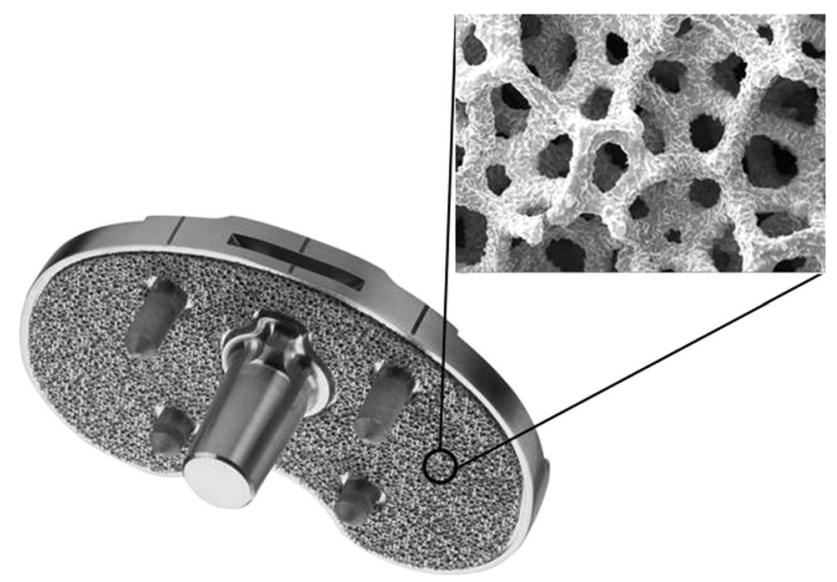

Fig. 1 ADVANCE ${ }^{\circledR}$ BIOFOAM $^{\circledR}$ tibial component is shown. The $100 \times$ magnification displays the roughened and porous surface of BIOFOAM. These components feature a Morse taper that allows for the attachment of various stem options

\section{Materials and methods}

One hundred and four (104) TKAs were performed in 85 subjects using the BIOFOAM-coated tibial components. Implantations were performed at four surgical centers by four surgeons. Institutional review board approval was obtained at each site prior to the enrollment of any subjects. Subjects were included if they required a TKA, were considered skeletally mature, and were considered to have a life expectancy to exceed 2 years. Subjects were excluded if they had a body mass index (BMI) greater than 45 , an active infection, or were pregnant. All tibial components were implanted with cementless fixation, and femoral component fixation was at the discretion of the implanting surgeon. The patella was resurfaced in $26.0 \%$ of TKAs, all using cemented patellar components. TKAs were performed using the subvastus $(70.2 \%)$, medial parapatellar (28.8\%), or midvastus $(0.9 \%)$ surgical techniques. The mean tourniquet time was $42.9 \mathrm{~min}$ (range $23.0-125.0 \mathrm{~min}$ ). Demographics for included subjects are listed in Table 1.

Subjects were evaluated preoperatively, and at 6,12 , and 24 months postoperatively. Knee Society Scores (total and function) and maximum flexion were used to assess functional outcomes [9]. Anterior-posterior (AP), lateral, and sunrise radiographs were also collected at each postoperative interval. Radiographs were reviewed for the

Table 1 Demographics for included subjects

\begin{tabular}{ll}
\hline Age & 64 years (range 43.0-87.0) \\
Male/female & $44(42.3 \%) / 60(57.7 \%)$ \\
BMI & $32.0 \mathrm{~kg} / \mathrm{m}^{2}\left(\right.$ range $\left.22.8-44.7 \mathrm{~kg} / \mathrm{m}^{2}\right)$ \\
$\begin{array}{l}\text { Primary indications } \\
\text { Degenerative osteoarthritis }\end{array}$ & $102(98.1 \%)$ \\
Avascular necrosis & $2(1.9 \%)$ \\
\hline
\end{tabular}

number and size of radiolucencies in the 17 zones shown in Fig. 2 and for the evaluation of patella tracking.

\section{Statistical analysis}

Knee Society Scores and maximum flexion at each interval were reported using mean and standard deviation values. These values were compared to baseline using a paired $t$ test $(p<0.05)$. Radiolucencies were reported as the number and mean size occurring in each zone. All statistical analyses were performed using SAS version 9.1.3 (SAS Institute Inc., Cary, NC).

\section{Results}

At each follow-up interval, Knee Society Scores (total and function) were significantly improved from baseline (Table 2). Flexion was significantly improved at 12 and 24 months. Representative radiographs of a well-fixed BIOFOAM tibial component are shown in Fig. 3. One subject experienced a 1-mm radiolucency in Zone 14 at 24 months. No other subjects presented with tibial zone radiolucencies. None of the tibial radiolucencies were progressive, and there were no signs of loosening or subsidence in either group. Six (6) subjects had radiolucencies in femoral zones at 24 months: 4 subjects with a radiolucency in Zone 1 (average size of $1.25 \mathrm{~mm}$ ); 1 subject with a $1-\mathrm{mm}$ radiolucency in Zone 5; and 1 subject with 1-mm radiolucencies in Zones 6 and 7, respectively. Radiographic analysis of the patella showed $94.0 \%$ (100\% of resurfaced patellae and $91.7 \%$ of non-resurfaced patellae) were tracking normally at 24 months. Of the five patellae not tracking normally, three were tilted and two were subluxed.

There were two revisions in the cohort. One TKA was revised at 2 months due to instability following a ruptured lateral collateral ligament (LCL). Another was revised at 16 months for recurrent dislocation of the tibial insert. This subject also experienced several complications prior to revision including: pain at 5.7 months, displacement and revision of the tibial insert at 5.8 months, and a stress fracture of the fifth metatarsal at 7 months. Other complications for the cohort included: one hematoma, one case of unexplained pain, one foot drop, and one decreased range of motion treated with manipulation under anesthesia. There were no intraoperative complications.

\section{Discussion}

Aseptic loosening remains one of the most common indications for revision TKA, accounting for over $30 \%$ of these procedures performed in the UK during 2012 [2]. 
Fig. 2 Tibial component radiographic zones used to evaluate radiolucencies

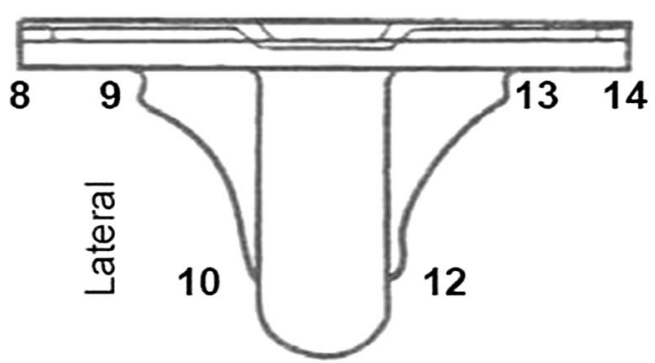

11

A-P View

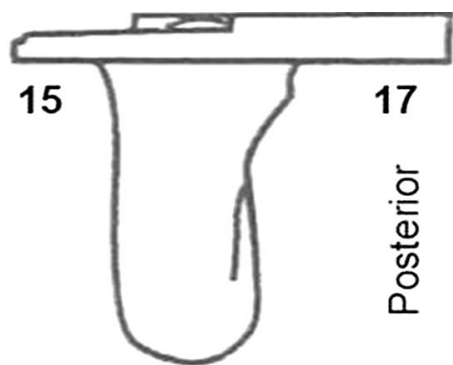

16

Lateral View
Table 2 Mean Knee Society Scores and range of motion values at each follow-up interval

\begin{tabular}{lcc}
\hline & Mean \pm Std. dev. & $p$ value \\
\hline Knee Society Scores & & \\
Preoperative & $53.6 \pm 15.2$ & - \\
6 months & $87.2 \pm 14.3^{*}$ & $<0.001$ \\
12 months & $90.0 \pm 10.3^{*}$ & $<0.001$ \\
24 months & $93.3 \pm 10.0^{*}$ & $<0.001$ \\
Knee Society Function Scores & \\
Preoperative & $52.6 \pm 15.2$ & - \\
6 months & $83.9 \pm 17.0^{*}$ & $<0.001$ \\
12 months & $87.0 \pm 16.3^{*}$ & $<0.001$ \\
24 months & $87.5 \pm 18.5^{*}$ & \\
Maximum flexion $\left(^{\circ}\right)$ & & - \\
Preoperative & $110.3 \pm 15.1$ & 0.001 \\
6 months & $111.5 \pm 13.1$ & 0.008 \\
12 months & $114.8 \pm 11.4^{*}$ & $<0.001$ \\
24 months & $116.8 \pm 11.4^{*}$ & \\
\hline
\end{tabular}

* Represents a statistically significant difference from preoperative values $(p=0.05)$

TKA with cemented fixation continues to be popular because it can reduce the risk of early loosening, but it does have some potential drawbacks compared to cementless fixation including increased operating/tourniquet time [10] and increased risk of thromboembolism [11]. Because aseptic loosening persists as a failure mode and to gain the benefits of cementless fixation, various surface finishes and component designs have been developed over the past several decades. One such technology is the BIOFOAMcoated tibial component.

BIOFOAM has a porosity of up to $70 \%$, which is significantly higher than that measured in titanium porous bead coatings [12]. A previous animal study compared bone ingrowth present in cylinders coated with either BIOFOAM- or titanium-sintered beads implanted in the diaphyses of nine dogs [7]. At 12-week follow-up, histological analysis showed there was more bone present in the BIOFOAM-coated specimens than there was space available for bone ingrowth in the porous bead specimens. BIOFOAM has a coefficient of friction that is over twice that of titanium porous bead coatings and nearly double that of titanium plasma spray coatings, which suggests that BIOFOAM is capable of providing increased resistance to motion prior to bone ingrowth when compared to other surface coatings [12]. While these characteristics make the material ideal for enhancing both short- and long-term implant fixation, clinical outcomes for these components have not been previously reported. In the current study, there were no complications or revisions related to migration or aseptic loosening, potentially supporting these described theoretical advantages.

BIOFOAM also has a compressive modulus similar to that of native bone, which may lead to more natural load transfers and reductions in stress shielding as previously described [7]. In the current study, only a single subject $(1.2 \%)$ presented with a radiolucency surrounding a BIOFOAM tibial component. Mean postoperative Knee Society Scores were excellent at final follow-up and similar to values previously reported in the literature for TKA. Revisions and complications were rare, and there were no failures attributable to the use of the BIOFOAM material or that have not been reported for other implant designs and coatings.

\section{Limitations}

The main limitations of the current study are the relatively small sample size and the duration of follow-up. None of the observed radiolucencies progressed during early study visits, but it is possible they could during later follow-up. There is also the potential for the development of new radiolucencies over time.

\section{Conclusions}

In conclusion, this preliminary study shows promising evidence for the use of BIOFOAM-coated tibial components in cementless TKA. Only a single subject 
Fig. 3 Representative anteriorposterior and lateral radiographs of a well-fixed BIOFOAMcoated tibial component
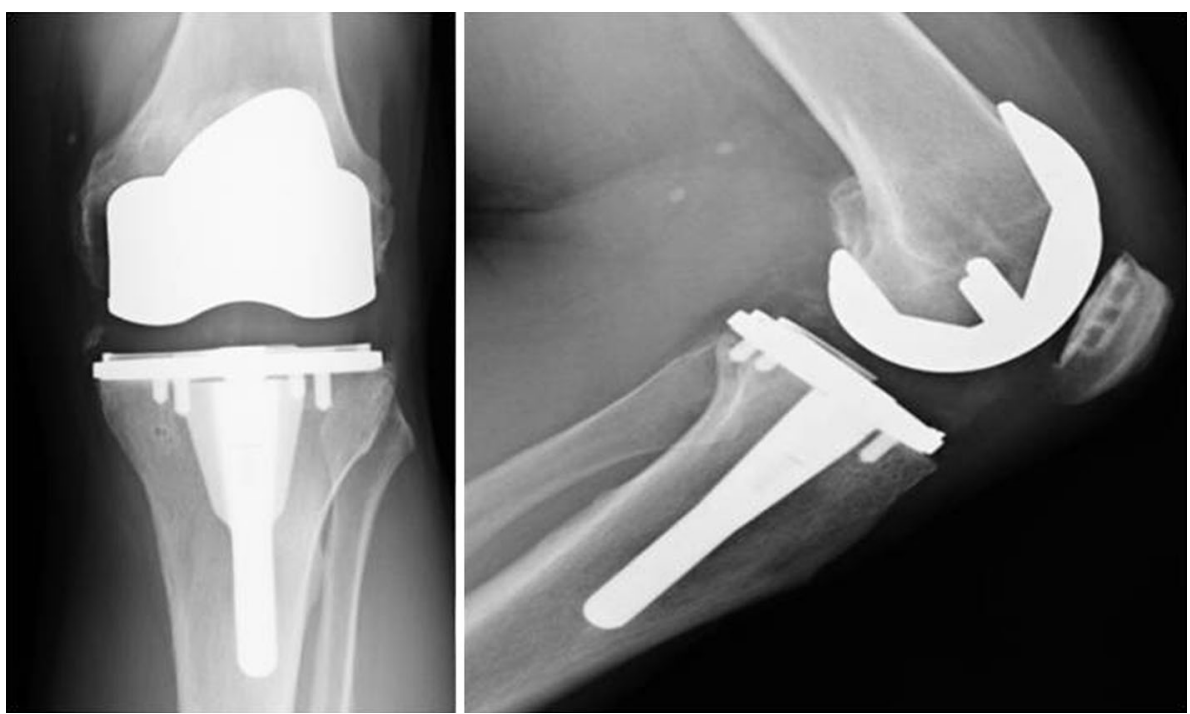

experienced a radiolucency surrounding a tibial component, and functional outcomes were excellent. Further investigations are needed to confirm whether these positive early results continue in the long-term and whether outcomes for these components differ from other fixation methods.

\section{Compliance with ethical standards}

Conflict of interest One author is a paid consultant of MicroPort Orthopedics Inc., and three authors are paid employees of MicroPort Orthopedics Inc.

Open Access This article is distributed under the terms of the Creative Commons Attribution 4.0 International License (http://crea tivecommons.org/licenses/by/4.0/), which permits unrestricted use, distribution, and reproduction in any medium, provided you give appropriate credit to the original author(s) and the source, provide a link to the Creative Commons license, and indicate if changes were made.

\section{References}

1. Danish Knee Arthroplasty Register-2013 Annual Report (2013). http://www.dkar.dk/. Accessed 9 Mar 2014

2. The 10th Annual Report of the National Joint Registry for England, Wales, and Northern Ireland (2013). http://www.njrcentre. org.uk/. Accessed 9 Mar 2014

3. Chinzei N, Ishida K, Tsumura N, Matsumoto T, Kitagawa A, Iguchi T, Nishida K, Akisue T, Kuroda R, Kurosaka M (2014) Satisfactory results at 8 years mean follow-up after ADVANCE(R) medial-pivot total knee arthroplasty. Knee 21(2):387-390. doi:10.1016/j.knee.2013.10.005

4. Karachalios T, Roidis N, Giotikas D, Bargiotas K, Varitimidis S, Malizos KN (2009) A mid-term clinical outcome study of the
Advance Medial Pivot knee arthroplasty. Knee 16(6):484-488. doi:10.1016/j.knee.2009.03.002

5. Schmidt R, Ogden S, Blaha JD, Alexander A, Fitch DA, Barnes CL (2014) Midterm clinical and radiographic results of the medial pivot total knee system. Int Orthop 38(12):2495-2498. doi:10.1007/s00264-014-2444-5

6. Vecchini E, Christodoulidis A, Magnan B, Ricci M, Regis D, Bartolozzi P (2012) Clinical and radiologic outcomes of total knee arthroplasty using the Advance Medial Pivot prosthesis. A mean 7 years follow-up. Knee 19(6):851-855. doi:10.1016/j. knee.2012.04.002

7. Scholvin D OR, Moseley J, Carroll M, Urban R, Turner T, Hall D, Waddell D, Rasmussen L (2008) Highly porous reticulated titanium foam for orthopaedic implant applications. In: Paper presented at the AAOS, San Fransico, CA

8. Abu-Rajab RB, Watson WS, Walker B, Roberts J, Gallacher SJ, Meek RM (2006) Peri-prosthetic bone mineral density after total knee arthroplasty. Cemented versus cementless fixation. J Bone Joint Surg $\mathrm{Br}$ 88(5):606-613. doi:10.1302/0301-620X.88B5. 16893

9. Insall JN, Dorr LD, Scott RD, Scott WN (1989) Rationale of the Knee Society clinical rating system. Clin Orthop Relat Res 248:13-14

10. Gandhi R, Tsvetkov D, Davey JR, Mahomed NN (2009) Survival and clinical function of cemented and uncemented prostheses in total knee replacement: a meta-analysis. J Bone Joint Surg Br 91(7):889-895. doi:10.1302/0301-620X.91B7.21702

11. Zhang ZH, Shen B, Yang J, Zhou ZK, Kang PD, Pei FX (2015) Risk factors for venous thromboembolism of total hip arthroplasty and total knee arthroplasty: a systematic review of evidences in ten years. BMC Musculoskelet Disord 16:24. doi:10. 1186/s12891-015-0470-0

12. Obert RSD, Carroll M (2007) Abrasion and frictional properties of a porous titanium foam. In: Society for biomaterials, Chicago, IL 